\title{
Three-Dimensional Arrangement and Connectivity of Lead-Chalcogenide Nanoparticle Assemblies for Next Generation Photovoltaics
}

\author{
Benjamin H. Savitzky ${ }^{1}$, Kevin Whitham ${ }^{2}$, Kaifu Bian ${ }^{3}$, Robert Hovden ${ }^{4}$, Tobias Hanrath ${ }^{3}$ and Lena F. \\ Kourkoutis ${ }^{4,5}$ \\ 1. Department of Physics, Cornell University, Ithaca, NY 14853, USA \\ 2. Department of Materials Science and Engineering, Cornell University, Ithaca, NY 14853, USA \\ 3. School of Chemical and Biomolecular Engineering, Cornell University, Ithaca, NY 14853, USA \\ 4. School of Applied and Engineering Physics, Cornell University, Ithaca, NY 14853, USA \\ ${ }^{5 .}$ Kavli Institute for Nanoscale Science, Cornell University, Ithaca, NY 14853, USA
}

The possibility of independently tuning electronic and optical properties of nanoparticle (NP) assemblies using inexpensive solution processing methods holds tremendous potential for scalable, efficient photovoltaic (PV) devices. In recent studies, “confined-but-connected" PbSe nanoparticle solids (NPSs) have been synthesized in which partial fusing of NPs in the superlattice results in dramatically increased electrical conductivity, while simultaneously preserving optical confinement. These NPSs have successfully been incorporated into a functioning proof-of-concept PV device, albeit at low efficiency [1]. Achieving and optimizing this combination of optoelectronic properties requires understanding and fine-tuning the material's nanoscale connectivity and three-dimensional (3D) structure.

TEM is a powerful tool that allows us to study the atomic scale structure of these NP systems as well as the 3D arrangement of individual NPs within their network through tomography. Here, we analyze two lead-chalcogenide NPSs. We show that the superlattice connectivity in PbSe NP assemblies depends on the NP shape and size, and illustrate alignment of the atomic lattice with the superlattice in connected samples. To understand the effect of applied pressure on the formation of NPSs, we use electron tomography to identify $3 \mathrm{D}$ ordering in $\mathrm{PbS} \mathrm{NP}$ assemblies.

Ordering in two PbSe NP assemblies is characterized by STEM and TEM imaging in Fig. 1. Cubic superlattices are formed with $6.9 \mathrm{~nm}$ diameter particles (Fig. 1(a,b)), and hexagonal ordering is observed in superlattices of $4.4 \mathrm{~nm}$ diameter NPs (Fig. 1(c,d)). TEM imaging revealed partial NP fusion in the cubic NP lattice sample resulting in continuous atomic lattice across multiple particles, indicating a NPS with good connectivity, while such connectivity was lacking in the hexagonal NP sample. Alignment of the atomic lattice with the superlattice for the $6.9 \mathrm{~nm}$ particles is also evident in the FFT of a superlattice grain, in which clear atomic lattice spots lie on axis with superlattice spots (left inset in Fig. 1(b)). In contrast, the $4.4 \mathrm{~nm} \mathrm{NP}$ atomic lattices orient at random, yielding hexagonal superlattice spots but atomic lattice rings in the FFT, shown in Fig. 1(d).

Optoelectroic properties in NPSs have also been shown to be highly dependent on the NP's 3D regimentation [2]. Electron tomography allowed us to determine the $3 \mathrm{D}$ structure of a $\mathrm{PbS}$ NPS, pressure treated at $15 \mathrm{GPa}$ to induce partial connectivity. 3D ordering is evident in Fig. 2, through two different zone axes $(a, b)$, and a slice through the reconstruction (c). The HAADF STEM tilt series was acquired from $-60^{\circ}$ to $75^{\circ}$, at $1.5^{\circ}$ increments, then reconstructed using a 30 iteration SIRT algorithm.

[1] W. J. Baumgardner, K. Whitham, and T. Hanrath, Nano Lett. 13 (2013), 3225-3231

[2] O. L. Lazarenkova, and A. A. Balandin, J. App. Phys. 89 (2001), 5509-5515 
[3] BHS acknowledge support from the NSF IGERT program (DGE-0903653). The CCMR Shared EM facility is supported through the NSF MRSEC program (DMR-1120296).
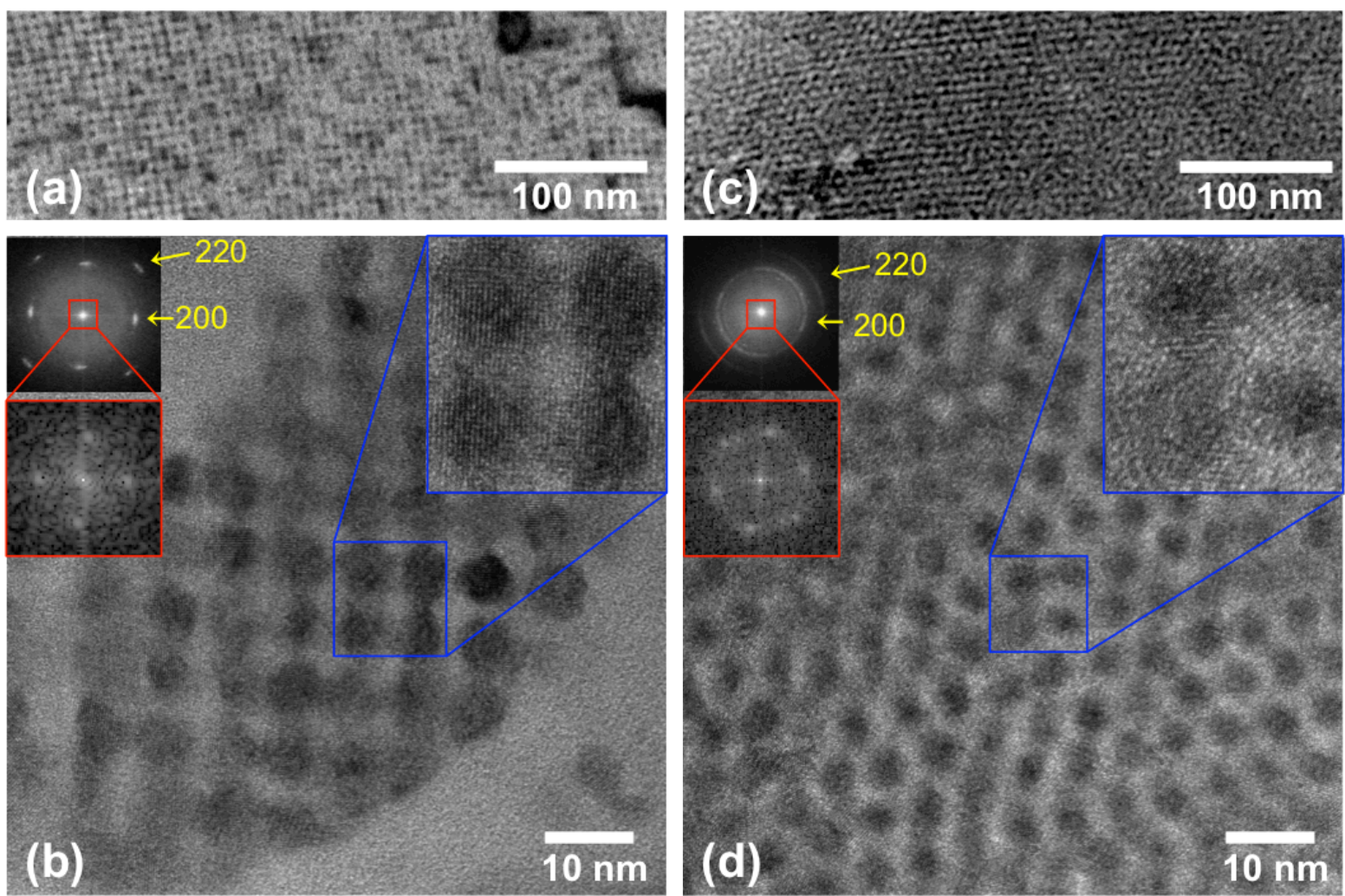

Figure 1. PbSe nanoparticle (NP) assemblies, forming a square superlattice from $6.9 \mathrm{~nm}$ diameter NPs $(\mathrm{a}, \mathrm{b})$ and a hexagonal superlattice from $4.4 \mathrm{~nm}$ diameter NPs (c, d). The atomic lattice fringes (right insets) in individual NPs are aligned with the superlattice in (b) but not in (d). The FFTs (left insets) show the periodicity of both the superlattices (bottom) and atomic lattices (top), confirming their alignment in the square but not in the hexagonal superlattice.

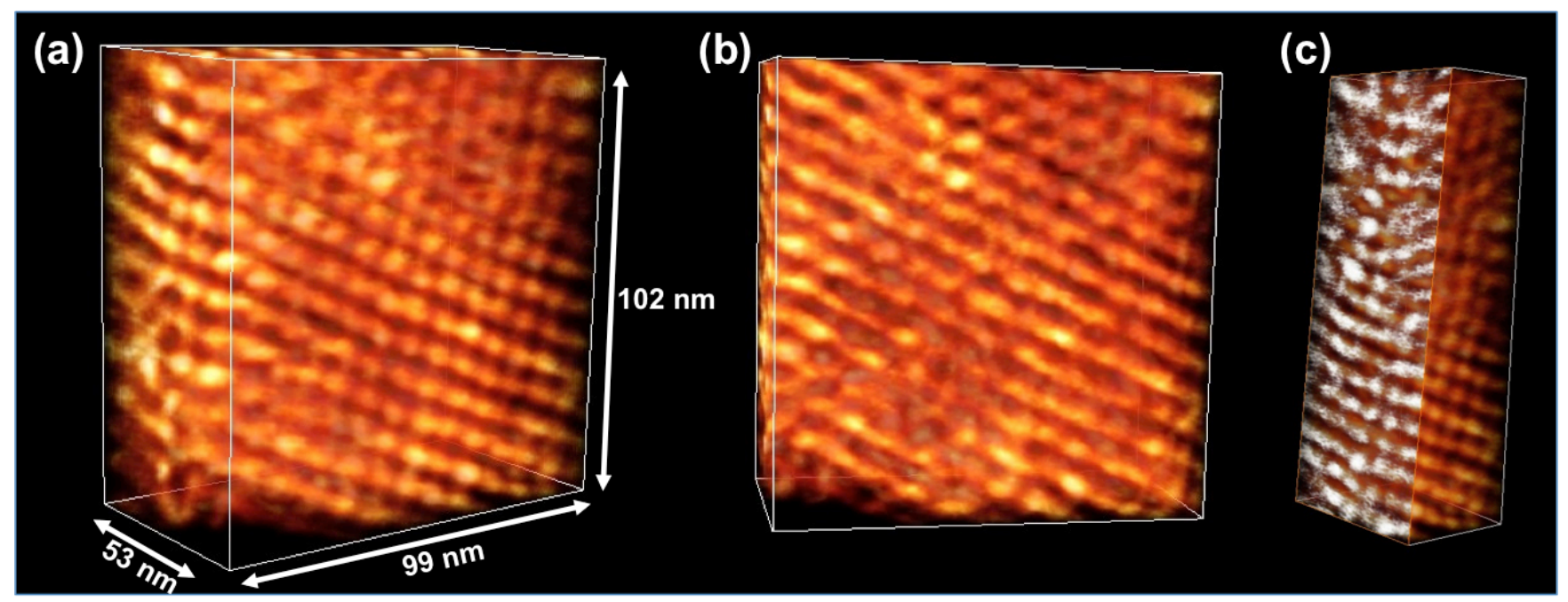

Figure 2. Tomographic reconstruction of a self assembled $\mathrm{PbS}$ nanoparticle (NP) superlattice, pressure treated at $15 \mathrm{GPa}$, illustrating three-dimensional NP ordering. The 3D structure is shown by direct volume rendering of the reconstruction. A single slice through the reconstruction is highlighted in (c). 